\title{
Analysis of Economic Disparities in Beijing-Tianjin-Hebei Region Based on Theil Index
}

\author{
Qingmin Yuan ${ }^{a}$, Shilei Liu ${ }^{b}$ \\ School of Management, Tianjin University of Technology, Tianjin 300384, China. \\ a15822363352@163.com, bliushilei508@163.com
}

Keywords: Beijing-Tianjin-Hebei; Theil index; Regional economic differences.

\begin{abstract}
Taking the population as the weight and the weight of income as indicators to measure the economic differences in the three major regions of the Beijing-Tianjin-Hebei region, and using the Theil index to describe the degree of economic disparity in the three regions. The results show that the degree of economic disparity among the three major regions of Beijing, Tianjin and Hebei is obvious, and the resource endowments, historical humanities and regional policies may be the main reasons for the obvious regional economic differences. Under the weighted comparison of population and income, there is no difference between the northern ecological area and the southern development area. The Theil index with the weight of income in the central core area is higher than the population weighted Theil index. At the end, there are targeted proposals and countermeasures to narrow regional economic disparities.
\end{abstract}

\section{Introduction}

In recent years, Beijing-Tianjin-Hebei region has developed rapid economic development, but there have also been some problems to be solved. It is mainly manifested in the economic development foundation of the central central region, the rapid growth of GDP and the large population flow. However, the economic development between the north and the south is not balanced, and the economic development between Beijing-Tianjin-Hebei has gradually widened. Domestic scholars use the Tel index to study regional differences more. Guo Yuanyuan and $\mathrm{Li} \mathrm{Li}$ used Gini coefficient, Theil index and variation coefficient to wake up and analyze the evolution of the 1994-2014 regional economic disparities in Chongqing, and to analyze [1]. Liu Fang and Wang Peixuan used multiple indicators to quantitatively measure the regional economic disparity in Jiangsu since 2002. We used absolute difference, relative difference, MLD index and Theil index to describe the degree of economic difference between the three regions, [2]. Zhang Yandong and Zhao Tao applied the Theil index to study the regional differences of energy consumption in China. In the two ways of three regions and richness zoning, we discussed the difference degree of similarity between energy consumption and economic growth, industry and population development respectively [3]. Some scholars have used a variety of methods to study the differences in regional income distribution and education expenditure and have obtained the corresponding results [4-6]. This paper studies the differences of regional economic development in Beijing, Tianjin and Hebei, finds out the forms and causes of regional economic development, and puts forward targeted countermeasures to narrow regional economic development differences, which is of practical significance for promoting the coordinated development of economy in Beijing-Tianjin-Hebei region.

\section{Research Methods and Data Sources}

\subsection{Research Methods}

The study uses the Tel index to analyze the regional economic differences in 2005-2015 BeijingTianjin-Hebei region. If the weight of the population is weighted, the calculating formula of the Tel index is as shown below:

$$
T=\sum P_{i} \times \log \left(\frac{P_{i}}{G D P_{i}}\right)+\sum P_{i} \times T_{i}
$$




$$
T_{i}=\sum_{j} \frac{p i j}{p i} \times \log \left(\frac{p i j / p i}{g d p i j / g d p i}\right)
$$

$\mathrm{T}$ is overall Theil index, $\mathrm{i}$ is the number of groups, the $\mathrm{i}=3 . \mathrm{Pi}$ is the proportion of the population in the $i$ function area in the overall research area of Beijing-Tianjin-Hebei. GDPi is the proportion of GDP in the $i$ function area of Beijing, Tianjin and Hebei. pi represents the population of the $i$ functional zone; gdpij represents $\mathrm{j}$ City GDP in the $\mathrm{i}$ region; pij indicates the population of $\mathrm{j}$ in the $\mathrm{i}$ area. The $\mathrm{Ti}$ is the unweighted regional inner ring index.If the income is weighted, the calculation formula of the Tel index will be different. The specific algorithms are as follows:

$$
\begin{gathered}
T=\sum P_{i} \times \log \left(\frac{G D P_{i}}{P_{i}}\right)+\sum G D P_{i} \times T_{i} \\
T_{i}=\sum_{j} \frac{g d p i j}{g d p i} \times \log \left(\frac{g d p i j / g d p i}{p i j / p i}\right)
\end{gathered}
$$

\subsection{Data Sources}

In the process of research, we divide the Beijing Tianjin Hebei region into three functional areas according to its functions: the central core area, the northern ecological area and the southern development area. Among them, Beijing, Tianjin, Baoding Tangshan and Langfang are the core functions of the central part in the traditional sense. The northern ecological areas include Zhangjiakou, Chengde and Qinhuangdao. The southern expansion areas include Shijiazhuang, Handan, Xingtai, Cangzhou and Hengshui. This study takes 2005-2015 as the study time and takes 13 cities in Beijing-Tianjin-Hebei as the research object. The data of the total population of the 13 cities in the Beijing-Tianjin-Hebei region (10000 people) and GDP (100 million yuan) are from the China Statistical Yearbook (2006-2016).

\section{Results and Analysis}

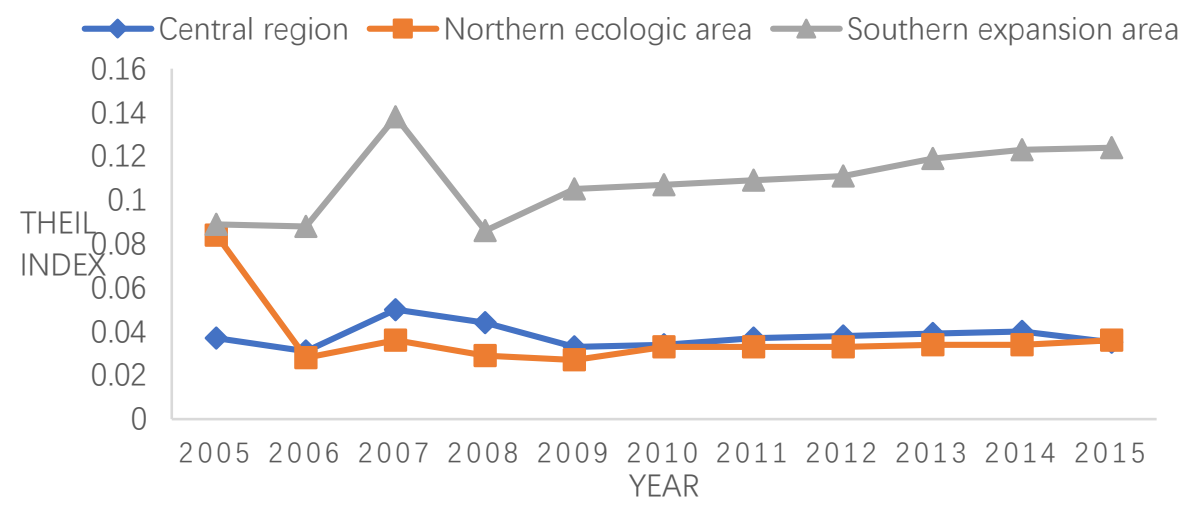

Figure.1. 2005-2015 years of economic disparity in the region of Beijing-Tianjin-Hebei with the weight of population

From the Tel index with the weight of population in Figure 1, we can see that the southern expansion area > the central core area > the northern ecologic area. It shows that the economic difference in the southern expansion area is the biggest and the development is the most unbalanced. The central and northern parts are basically the same, and the north is slightly less. It shows that although the three cities in the north are not high at the level of economic development, the development trend is basically balanced. The central economic development is the best, but there is a certain gap between the three cities outside Beijing and Tianjin and the two cities of Beijing and Tianjin. As a whole, the three areas have been obviously rising in 2006-2007 years, and there is a significant decline in the 2007-2008 years. Among them, the southern expansion area has a slow growth trend in 2008-2015 years, and the regional economic disparity of population is more obvious. In 2007, Theil index reached the maximum value of 0.138 . The central and northern parts are basically flat, and the economic development of the population is more uniform. 


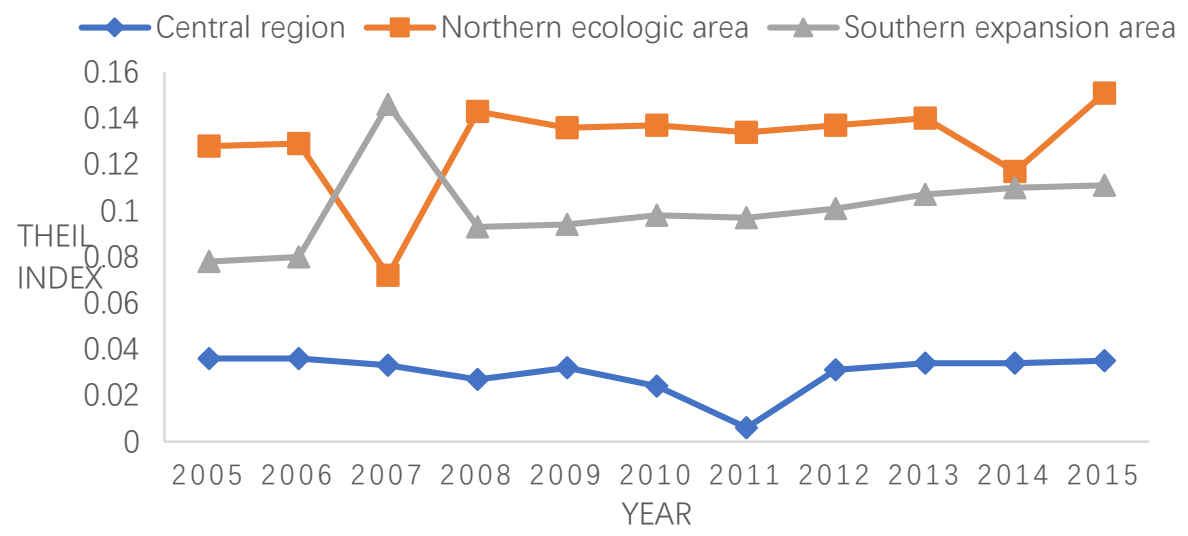

Figure.2. 2005-2015 years of economic disparity in the region of Beijing-Tianjin-Hebei with the weight of the income as the weight

From the Tel index of income as the weight of Figure 2, we can see that the central region of the central region > South expansion area > the northern ecologic area. In the case of income, the economic development of the central region is the biggest difference, the South expansion area is the second, the northern ecologic area is the smallest. The central core area's Theil index has a significant decline in 2006-2007 years and 2013-2014 years. It shows that in 2007-2014 years, the difference of economic development in the region is narrowing while considering the income, and the economic difference in 2008-2013 years is relatively stable. The 2007 Tel index reached a minimum of 0.072 . The development trend of the southern development area increased significantly in the past 20062007 years, reaching a maximum value of 0.146 in 2007, while in 2008-2015 years, it was rising slowly. The northern ecological region was in a slow decline in 2005-20011, which reached a minimum of 0.006 in 2011.

\section{Conclusion}

The main reasons for the differences in economic development in Beijing- Tianjin-Hebei may be related to geographical location, historical humanities and regional policies. The central region of the central region has a good industrial base, which has the advantages of policy, traffic, history and culture. This plays an important role in attracting talent and capital inflow. The three cities in the northern ecological area are mostly mountain areas, so it is determined that the traffic is limited and the people are not very much. The natural resources of the southern expansion area are relatively scattered, and the advantages of the policy are not obvious. The coordinated development of BeijingTianjin-Hebei should break the administrative regional restrictions, share the resources, divide the division of labor and cooperate with each other. The coastal cities should make use of the advantages of the port and the marine resources, and the mountain cities should pay more attention to the development of ecotourism. This can gradually reduce the economic differences between regions and promote the rational development of regional economy.

\section{References}

[1]. Guo Yuan Yuan, Li Li. The influence factors of regional economic differences in the western inland provinces - a case study of [J]. geography in Chongqing, 2017,36 (05): 926-944.

[2]. Liu Fang, Wang Peixuan. Analysis of regional economic differences based on the Tel index: a case of Jiangsu Province as an example of [J]. regional economic review, 2013 (06): 103-108.

[3]. Zhang Yandong, Zhao Tao. Research on regional differences in energy consumption based on the Tel index [J]. arid region resources and environment, 2015,29 (06): 14-19.

[4]. Lin. Analysis on regional economic disparity Theil index -- Taking the Yangtze River economic belt as an example based on the [J]. (on a), 2015 (07): 50-55. 
[5]. Yang Wen, Wang Haimin. Analysis of the regional differences in financial expenditure on financial education in China [J]. financial problems, 2014 (05): 79-84.

[6]. Peng Dingyun, Chen Weiyi. The consumption gap of the Theil index on the income distribution based on [J]. Journal of Zhongnan University of Economics and Law, 2014 (02): 30-37. 